\title{
La estrogenoterapia de reemplazo no fue efectiva en la enfermedad de Alzheimer
}

Estrogen Replacement Therapy for Treatment of Mild to Moderate Alzheimer's Disease. Mulnard RA, Cotman CW, Kawas C, et al. JAMA 2000; 283:1007-15

\section{Objetivo}

Determinar si la terapia de reemplazo hormonal (TRH) afecta la función cognitiva, la memoria y el deterioro funcional en mujeres con enfermedad de Alzheimer (EA) leve a moderada.

\section{Diseño}

Estudio prospectivo, aleatorizado, doble ciego, controlado.

Lugar

Treinta y dos sitios de estudio en EE.UU.

\section{Participantes}

Un total de 120 mujeres histerectomizadas con EA leve a moderada y un Minimental (MMSE) entre 12 y 28. Un año de seguimiento.

\section{Intervenciones}

Las participantes fueron distribuidas aleatoriamente en tres ramas: estrógenos $(E) 0,625 \mathrm{mg} / \mathrm{d}(n=42), 1,25 \mathrm{mg} / \mathrm{d}(\mathrm{n}=39)$, o placebo $(P)(n=39)$.

\section{Medición de los Resultados Principales}

Se evaluó la impresión clínica del cambio global (ICCG), que mide los cambios en una escala de siete puntos, en donde 4 es sin cambio, 7 es que empeoró mucho, y 1 que mejoró mucho. También se utilizaron puntajes previamente validados para evaluar el deterioro cognitivo como el ADAS-CogS1, así como el MMSE, la escala de depresión de Hamilton, el Clinical Dementia Rating Scale (CDR) y el Blessed para evaluación funcional.

\section{Resultados Principales}

Las características demográficas entre los grupos al inicio del protocolo no mostraron diferencias significativas. Completaron el estudio 97 pacientes. El análisis fue por intención de tratar*.

En la comparación de dos grupos (P vs E a baja y alta dosis) a los 12 meses no mostró diferencias significativas. El puntaje de ICCG para los pacientes con $E$ fue de 5.1 vs. 5.0 el grupo $P$ (pNS). El 80 $\%$ de los que tomaron E empeoraron en dicha evaluación vs el $74 \%$ de los que tomaban placebo ( $p$ NS) Ver tabla. Solo se observó una diferencia que sugería un leve empeoramiento en los tomadores de $E$ en el CDR.

\begin{tabular}{l|c|c}
\hline Resultados & Grupo Placebo (n=39) & Grupo Estrógeno (n=81) \\
\hline $\begin{array}{l}\text { Pacientes que empeoraron } \\
\text { en el ICCG (n, \%) }\end{array}$ & $28(74)$ & $64(80)$ \\
\begin{tabular}{l} 
Puntaje (media, DS) \\
\hline $\begin{array}{l}\text { Cambio en MMSE } \\
\text { (media, DS) }\end{array}$
\end{tabular} & $5.0(1.1)$ & $5.1(0.9)$ \\
\hline $\begin{array}{l}\text { Cambio en CDR } \\
\text { (media, DS) }\end{array}$ & $-3.1(4.1)$ & $-2.7(3.7)$ \\
\hline $\begin{array}{l}\text { Cambio en ADAS-Cog } \\
\text { (media, DS) }\end{array}$ & $0.2(0.4)$ & $0.5(0.6)$ \\
\hline
\end{tabular}

Todas las diferencias fueron no significativas, excepto el puntaje de CDR, que fue algo mejor en el grupo placebo.

\section{Conclusión}

La TRH durante un año no impidió la progresión de la EA leve o moderada ni mejoró las funciones cognitivas, motoras, o de las actividades de la vida diaria. Este estudio no avala la utilización de la TRH como tratamiento de la EA.

\section{Comentario}

La TRH continua en "el tapete de la discusión" tanto para los médicos de atención primaria como para las pacientes. En la última década hemos asistido a diferentes tipos de evidencias de que la TRH posee efectos sobre los sistemas cardiovascularés2 $y$, como nos concierne en este comentario, sobre las funciones cerebrales como las cognitivas 3,4 y de la memoria, además de los consabidos sobre el sistema musculoesquelético. Estas evidencias nacen a partir de estudios en medicina experimental y de trabajos clínicos observacionales de cohortes*.

No obstante los estudios controlados que avalen esta tendencia son pocos. Cabe mencionar que el estudio HERS no mostró beneficios de la TRH en la enfermedad cardiovascular establecida5. La EA, caracterizada por la progresiva pérdida de la memoria y de las funciones cognitivas, afecta a más de 15 millones de personas en todo el mundo. Por su escasa letalidad (la sobrevida a la década de realizado el diagnóstico es habitual) la prevalencia oscila desde el $3 \%$ a los 65 años hasta el $47 \%$ a los 85 años 6 . Es notorio que las mujeres doblan a los hombres en cuanto a la prevalencia de la enfermedad, y esto pareciese no solo estar explicado por su mayor longevidad. Se ha sugerido que la caída brusca de los estrógenos en la post menopausia estaría implicada en este fenómeno. Paradójicamente los hombres mantienen sus niveles de estrógenos cerebrales gracias a poseer la enzima que aromatiza la testosterona en estrógenos. Además existe evi- dencia desde la medicina experimental y convincentes razones biológicas para pensar que los $\mathrm{E}$ podrían influir en las funciones cognitivas en la postmenopausia.

Este meticuloso estudio (por ejemplo: cada paciente poseía un exclusivo supervisor que controlaba que realmente tomara la medicación y se realizaban dosajes plasmáticos de $\mathrm{E}$ a cada paciente en cada evaluación) muestra de manera clara e inequívoca que los $E$ no mejoran la EA declarada.

Recordemos que las metas que uno se propone al tratar le EA son mejorar 0 al menos retrasar la pérdida de la memoria y de otras funciones cognitivas y mantener la independencia y capacidades funcionales.

Ninguno de los múltiples test o puntajes utilizados para objetivar lo antedicho mostró algún cambio positivo o menos progresión de enfermedad. No obstante es de capital importancia entender, como señala el comentario editorial que lo acompaña8, que las conclusiones sólo son válidas para la población estudiada: paciente mujeres con EA declarada. Es decir no hay que extrapolar conclusiones acerca de su rol en la prevención primaria de la EA, que es otra línea en estudio.

Probablemente tendremos que esperar hasta el 2005 en que estarán los resultados del "Women's Health Initiative" en el que participan 64500 pacientes con un seguimiento de 14 años, para conocer el real efecto de los $E$ en prevención y/o tratamiento de la EA.

*Ver glosario

Dr. Manuel Montero-Odasso [ Medico Clínico y Geriatra. Vicedirector Carrera de Especialistas en Geriatría, UBA. Hospital Italiano de Buenos Aires ] Referencias

1. Leber P Draft guidelines for the clinical evaluation of antidementia drugs. Washington DC :Food and Drugs Administration, 1990 (FDA publicatin no. F91-19331

2. Grodstein D, et al. Postmenopausal estrogen and progestin use and the risk of carduiovascular disease. N.Eng.J. Med. 1996; 335: 453-461

3. Garret- D, Kritz-

3. Barret-Connor E, Kritz-SilversteinD. Estrogen replacement therapy and cognitive decline in

4. Barret-Conor E. Rethimking estrogen and the brain. J.Am.Geriat. Soc. 1998;46: 918-920
5 Hulley S, GradyD Bush T el al. Randomized trial estrogen plus progestin for secondaryof coronary heart disease in postmenopausal women. JAMA 1998; 280:605-613 (Estudio HERS)

5. Hulley S, GradyD Bush T el al. Randomized trial estrogen plus progestin for secondaryof coronary heart disease in postmenopausal women. JAMA

6. Herbert LE, Scherr PA, Beckett LA, et al. Age -specific incidence ogf Alzheimer's disease in a com
7. Mayeux, R and Sano, M. Treatment of Alzheimer's Disease. N EngJ Med 1999; 34: 1670-1679.

8. Shaywitz BA and Shawitz SE. Estrogen and Alzheimer's Diseases. Plausible Theory, Negative Clinical Trial JAMA 2000 283: 1055-1056 